\title{
Modelling parallel assemblies of porous materials using the equivalent circuit method
}

\author{
Reto Pieren ${ }^{\text {a) }}$ and Kurt Heutschi \\ Empa, Swiss Federal Laboratories for Materials Science and Technology, 8600 Duebendorf, \\ Switzerland \\ reto.pieren@empa.ch,kurt.heutschi@empa.ch
}

\begin{abstract}
Recently, the accuracy of the parallel transfer matrix method (P-TMM) and the admittance sum method (ASM) in the prediction of the absorption properties of parallel assemblies of materials was investigated [Verdière, Panneton, Elkoun, Dupont, and Leclaire, J. Acoust. Soc. Am. 136, EL90-EL95 (2014)]. It was demonstrated that $\mathrm{P}-\mathrm{TMM}$ is more versatile than ASM, as a larger variety of different backing configurations can be handled. Here it will be shown that the same universality is offered by the equivalent circuit method.

(C) 2015 Acoustical Society of America

[NX]

Date Received: September 2, 2014 Date Accepted: December 12, 2014
\end{abstract}

\section{Introduction}

A recent publication ${ }^{1}$ explored the applicability of the admittance sum method (ASM) and the parallel transfer matrix method ${ }^{2}$ (P-TMM) for the prediction of the absorption coefficient of parallel assemblies of materials. From comparisons with finite-element method calculations it was concluded that ASM only yields correct results if the backing is divided into separate cells and no acoustical leaks exist. In contrast, ("nonisolated") P-TMM also yields correct results for configurations featuring a common air volume backing. Here it will be shown that both backing configurations can be represented easily by a corresponding equivalent electrical network. The equivalent circuit method (ECM) turns out to be an alternative versatile approach and in this respect advantageous compared to ASM.

\section{Theory}

\subsection{Analog electrical networks}

Equivalent electrical networks of acoustical systems can be found by establishing an analogy between the acoustical quantities sound pressure $p$ and sound particle velocity $v$ or volume flow $q$ and the electrical quantities voltage $U$ and current $I$. In this article the $p U-q I$ analogy is adopted where the potential quantity $p$ is mapped onto $U$ and the flow quantity $q$ onto $I$. Once the analogy is established, fundamental structures in acoustical systems can be identified and described by corresponding electrical network elements.

\subsection{Fluid medium represented as $\Pi$-type circuit}

Acoustical structures that are small compared to the wavelength can be represented by lumped electrical elements. If the structures allow for sound propagation, they have to be represented by distributed elements. An important and often encountered distributed element is an extended tube-like configuration carrying a plane wave in the axial direction. The length of the element is assumed to be $d$, where $d$ is not small compared to the wavelength. Materials that can be approximated by an equivalent fluid such as air or rigid frame open cell porous materials can be described by ${ }^{3,4}$

\footnotetext{
${ }^{\text {a) }}$ Author to whom correspondence should be addressed.
} 


$$
\begin{gathered}
\check{p}_{1}=\cosh \left(j k_{c} d\right) \cdot \check{p}_{2}+Z_{c} \sinh \left(j k_{c} d\right) \cdot \check{v}_{2}, \\
Z_{c} \cdot \check{v}_{1}=\sinh \left(j k_{c} d\right) \cdot \check{p}_{2}+Z_{c} \cosh \left(j k_{c} d\right) \cdot \check{v}_{2},
\end{gathered}
$$

with the imaginary unit $j$, the characteristic impedance $Z_{c}$, and the wave number $k_{c}$ of the material and the sound pressure $\check{p}$ and the sound particle velocity $\check{v}$ as complex amplitude functions on both sides of the medium distinguished by indices 1 and 2 . In the equivalent circuit, such elements are represented by electrical transmission lines. For analytical evaluations, it is convenient to introduce them as four-poles [Fig. 1(a)].

Whereas the characteristic impedance $Z_{c}$ is defined as the ratio of sound pressure and particle velocity, the following impedances are defined as the ratio of sound pressure $p$ and volume flow $q$. It can be shown ${ }^{4}$ that a four-pole with the behavior of Eqs. (1) and (2) can be represented by a $\Pi$-type circuit with generalized impedances [Fig. 1(b)] that are given by

$$
\begin{gathered}
Z_{\alpha}=\frac{Z_{c}}{S} \sinh \left(j k_{c} d\right), \\
Z_{\beta}=\frac{Z_{c} \sinh \left(j k_{c} d\right)}{S \cosh \left(j k_{c} d\right)-1},
\end{gathered}
$$

with $S$ being the cross sectional area of the element. As an alternative to the $\Pi$-type circuit discussed above, a T-type circuit can be used instead. ${ }^{4}$

\subsection{Arrangements of $\Pi$-type circuits}

Now networks of four-poles are investigated. For simplicity analytical expressions are deduced for the case of two elements $A$ and $B$ only-however, they can be applied iteratively and thus easily generalized for multiple elements. Double-layer arrangements can be modeled by a serial connection of $\Pi$-type circuits as shown in Fig. 2(a). This connection type satisfies the continuity condition of pressure and volume flow at the boundaries, i.e., $\check{p}_{1}^{B}=\check{p}_{2}^{A}$ and $\check{q}_{1}^{B}=\check{q}_{2}^{A}$. In Ref. 5 it is shown that this connection type incorporates the well-known impedance transfer method that is often used for multilayer structures.

The boundary conditions for parallel assemblies of media as formulated in Refs. 1 and 2 are $\check{p}_{1}^{B}=\check{p}_{1}^{A}, \check{p}_{2}^{B}=\check{p}_{2}^{A}, \check{q}_{1}=\check{q}_{1}^{B}+\check{q}_{1}^{A}$, and $\check{q}_{2}=\check{q}_{2}^{B}+\check{q}_{2}^{A}$. These conditions imply a parallel connection of the corresponding $\Pi$-type circuits as depicted in Fig. 2(b). At the connections of the two elements, Kirchhoff's circuit law assures that the boundary conditions are met. Whereas in the serial arrangement case as depicted in Fig. 2(a), field quantity calculations can easily be handled, at first glance, the parallel arrangement as depicted in Fig. 2(b) provides more difficulties as the network contains multiple overlapping loops. However, this network can be transformed into an equivalent network as shown in Fig. 2(c). This representation is convenient to handle parallel

(a)

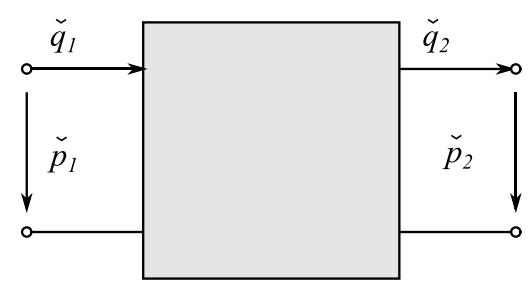

(b)

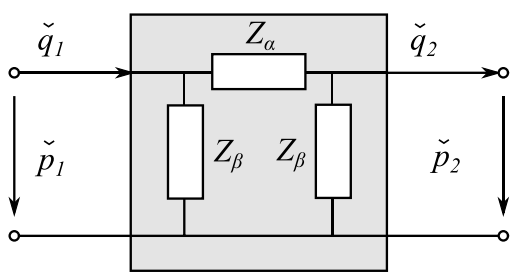

Fig. 1. General four-pole that establishes the relations between pressure and velocity at the two ports 1 and 2 (a) and a four-pole as a $\Pi$-type circuit of generalized impedances $Z_{\alpha}$ and $Z_{\beta}$ (b). 
(a)

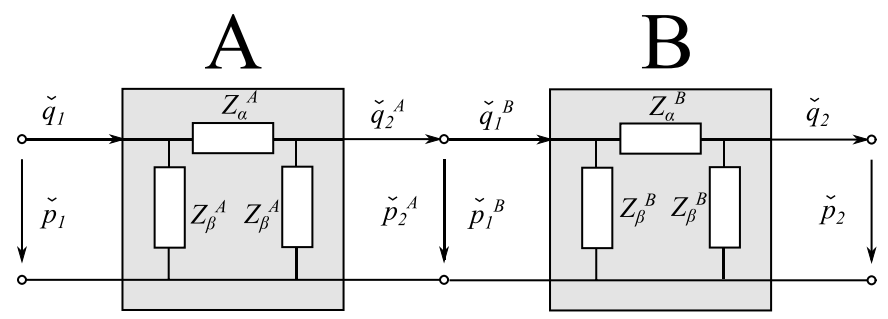

(b)

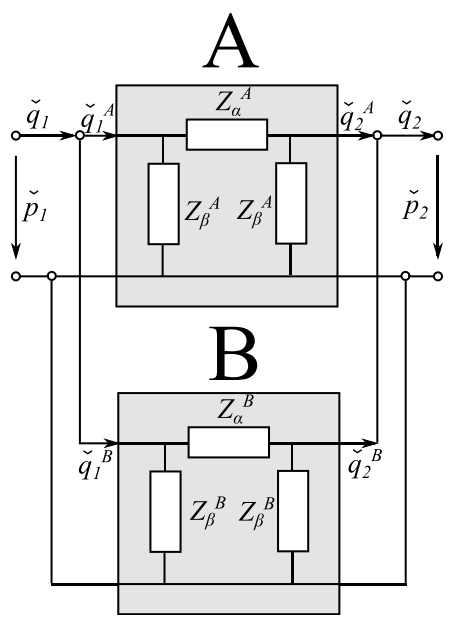

(c)

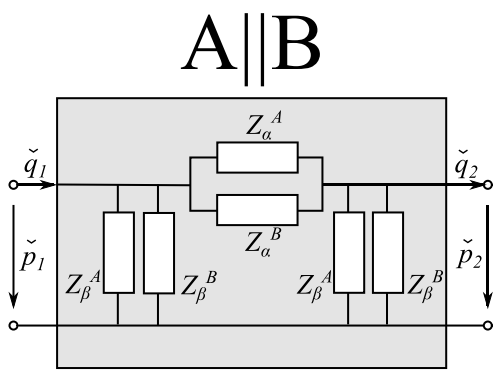

Fig. 2. Arrangements of П-type circuits. (a) Serial connection of two four-poles A and B representing a multilayer arrangement. (b) Parallel connection of two four-poles A and B representing a parallel patch arrangement and an equivalent representation (c).

connected two-ports analytically. By evaluation of this representation finally an equivalent $\Pi$-type circuit representing the parallel connection of $A$ and $B$ can be deduced:

$$
\begin{aligned}
& Z_{\alpha, A \| B}=Z_{\alpha}^{A} \| Z_{\alpha}^{B} \equiv\left[\frac{1}{Z_{\alpha}^{A}}+\frac{1}{Z_{\alpha}^{B}}\right]^{-1}, \\
& Z_{\beta, A \| B}=Z_{\beta}^{A} \| Z_{\beta}^{B} \equiv\left[\frac{1}{Z_{\beta}^{A}}+\frac{1}{Z_{\beta}^{B}}\right]^{-1},
\end{aligned}
$$

where $\|$ denotes the parallel connection of two impedances.

\subsection{Absorption coefficient}

The total cross sectional area of the assembly is $S_{\text {tot }}=\sum S_{i}$. For the calculation of the absorption coefficient only the relative cross sectional areas $r_{i}=S_{i} / S_{\text {tot }}$ of the cells are relevant. Thus by replacing $S$ in Eq. (3) and (4) by $r_{i}$, the volume flow quantities are converted into weighted particle velocities, i.e., $\breve{q}_{1} \rightarrow \breve{v}_{1}$. The equivalent circuit has to be terminated by a discrete impedance $Z_{t}$ representing the backing of the structure. For a hard backing, $Z_{t}=\infty$. For an anechoic backing as described in Ref. 1 and normal sound incidence follows $Z_{t}=Z_{0} / r_{t}$ with $Z_{0}=\rho_{0} c_{0}$ being the characteristic impedance of air with density $\rho_{0}$ and speed of sound $c_{0}$. With the help of the surface 
impedance (input impedance), $Z_{s}=\check{p}_{1} / \check{v}_{1}$, in the resulting equivalent circuit the normal incidence absorption coefficient is determined as

$$
\alpha_{0}=1-\left|\frac{Z_{s}-Z_{0}}{Z_{s}+Z_{0}}\right|^{2} \text {. }
$$

\section{Equivalent circuit method versus P-TMM and ASM}

To demonstrate the versatility of ECM, the six test cases discussed in Ref. 1 are numerically investigated by considering the equivalent circuit (see Fig. 3). The test cases can be subdivided into two types. In the case of type 1 geometries (left column in Fig. 3), each cell possesses its own "isolated" air volume on the rear side. On the other hand, type 2 geometries (right column in Fig. 3) feature a "non-isolated" backing zone with the three cells sharing the same air volume. These two backing types are intuitively converted into equivalent circuits as shown at the bottom of Fig. 3. The type 1 configuration is a parallel arrangement of three individual branches, each having its

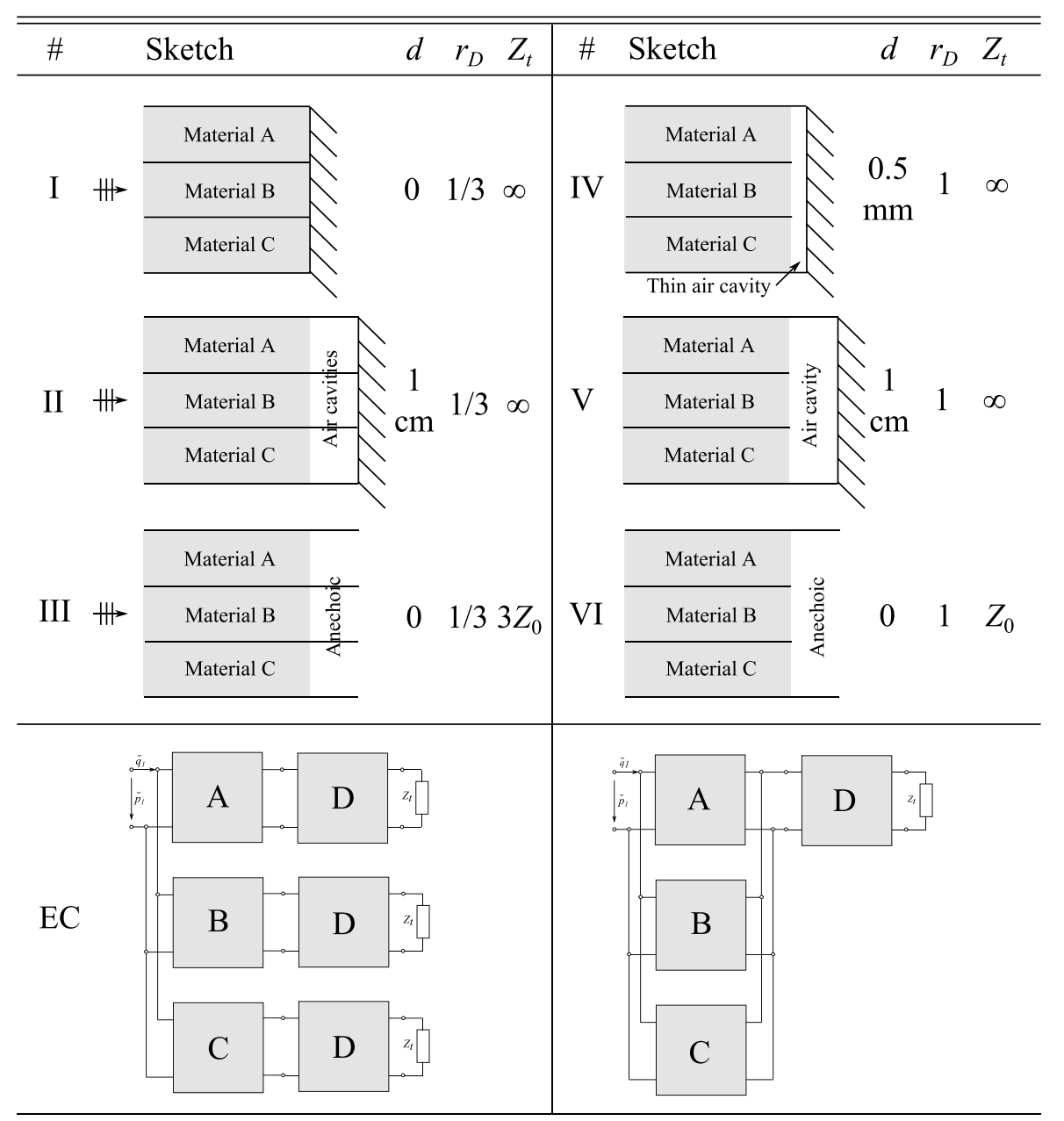

Fig. 3. Sketches of test cases I to VI from Ref. 1 and their corresponding equivalent circuits with the parameters describing the backing structure, i.e., the thickness $d$ and relative cross sectional area $r_{D}$ of the air cavity element $D$ and the termination impedance $Z_{t}$. 

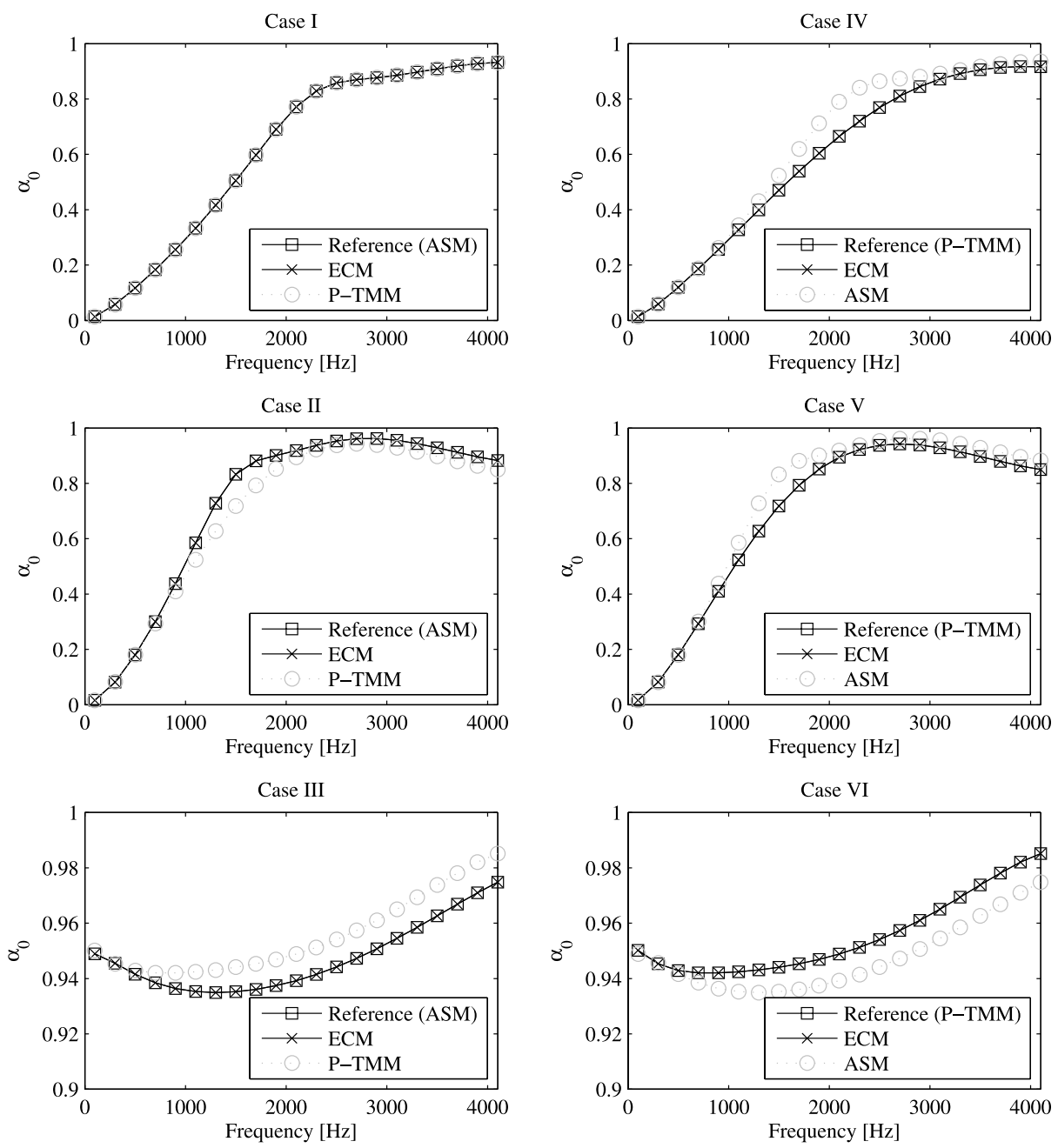

Fig. 4. Normal incidence sound absorption coefficients calculated by ASM, P-TMM, and ECM.

own termination, while the network of a type 2 backing merely configures the three cells in parallel but with a common termination.

Figure 4 shows the results for the absorption coefficient as obtained with ECM in comparison to ASM and P-TMM. The properties of the three materials are derived from the parameters specified in Ref. 1 with help of the Johnson-Champoux-Allard model. ${ }^{3}$ For type 1 backing configurations, ECM matches perfectly with ASM, for type 2 backings, ECM yields the same results as P-TMM.

\section{Conclusion}

By comparison with finite elements simulations, it was concluded in Ref. 1 that ASM is applicable for type 1 backing configurations only, while P-TMM can handle both backing types. In line with P-TMM, the numerical evaluations of ECM have demonstrated that the equivalent circuit method yields correct absorption coefficients in both backing conditions as well. Compared to ASM, ECM can be regarded as a more versatile approach to investigate the absorption properties of parallel assemblies of materials. 


\section{References and links}

${ }^{1}$ K. Verdière, R. Panneton, S. Elkoun, T. Dupont, and P. Leclaire, "Comparison between parallel transfer matrix method and admittance sum method,” J. Acoust. Soc. Am. 136(2), EL90-EL95 (2014).

${ }^{2}$ K. Verdière, R. Panneton, S. Elkoun, T. Dupont, and P. Leclaire, "Transfer matrix method applied to the parallel assembly of sound absorbing materials," J. Acoust. Soc. Am. 134(6), 4648-4658 (2013).

${ }^{3}$ J. F. Allard and N. Atalla, Propagation of Sound in Porous Media: Modelling Sound Absorbing Materials, 2nd ed. (Wiley, London, 2009), pp. 243-307.

${ }^{4}$ F. P. Mechel, Formulas of Acoustics, 2nd ed. (Springer, Berlin, 2008), pp. 64-70.

${ }^{5}$ R. Pieren and K. Heutschi, "Predicting sound absorption coefficients of lightweight multilayer curtains using the equivalent circuit method," Appl. Acoust. 92, 27-41 (2015). 